\title{
Large-Scale Convective Ekman Flow of Viscous Incompressible Fluid in the Equatorial Zone
}

\author{
A. V. Gorshkov ${ }^{1,2, a)}$ and E. Yu. Prosviryakov ${ }^{1,2, b)}$ \\ ${ }^{1}$ Institute of Engineering Science, Ural Branch of the Russian Academy of Sciences, \\ 34 Komsomolskaya St., Ekaterinburg, 620049, Russia \\ ${ }^{2}$ B. N. Yeltsin Ural Federal University, 19 Mira St., Ekaterinburg, 620002, Russian Federation \\ a)Corresponding author: e-mail alex55gor@mail.ru, \\ b) evgen_pros@mail.ru
}

\begin{abstract}
The paper studies the large-scale convective flow of viscous incompressible fluid in the equatorial zone, taking into account two components of the Coriolis force. Temperature gradients are set at the flow boundaries. The Marangoni effect works on the free boundary. The existence and position of stagnation points is analyzed. The parameter values at which stagnation points can appear are determined. The possibility of a double stagnation point is shown.
\end{abstract}

\section{INTRODUCTION}

The study of convective flows at the equator is one of the important problems of geophysical hydrodynamics. To date, the starting point of all investigations in geophysical hydrodynamics is the exact Ekman solution [1], which describes a stationary isobaric uniform rotation of a fluid in an infinite ocean. Generalizations of the Ekman shear flow are found in [2-12].

When calculating the world's ocean currents, the traditional Coriolis acceleration approximation is used, which is characterized by a single Coriolis parameter. Strictly speaking, this approach allows us to describe fluid motion at high latitudes $[4,6]$. As is known, this approach is also used for middle latitudes, since horizontal velocities in largescale currents exceed the vertical velocity by several orders of magnitude [4]. Despite these assumptions, integration of motion equations is a very difficult problem.

This paper offers exact solutions to the nonlinear Navier-Stokes equations describing fluid flows having a tilted axis of rotation. An important case is the tilt of the axis at $\pi / 2$. This case corresponds to a current near the equator.

\section{PROBLEM STATEMENT}

Large-scale laminar convective flow of viscous incompressible fluid in the equatorial zone is studied. It is assumed that the latitude of the place $\varphi$ is small and that it can be approximated by $\sin \varphi \approx 0, \cos \varphi \approx 1$. Let us introduce a local coordinate system as follows: the axis $o z$ is directed along the radius of the Earth; the axis $o y$ is tangent to the Meridian in the direction of the North Pole; the axis $o x$ is perpendicular to them and tangential to the equator.

In the local coordinate system $(x, y, z)$ the Earth's rotation axis is parallel to the axis $o y$. In what follows, we consider the shear flow of a viscous incompressible fluid, assuming that $V_{\mathrm{z}}=0$. The Oberbeck-Boussinesq equations describing the stationary large-scale Ekman flow of a heated inhomogeneous viscous incompressible fluid with two components of the Coriolis force in dimensionless variables take the form [9-17]

Mechanics, Resource and Diagnostics of Materials and Structures (MRDMS-2020)

AIP Conf. Proc. 2315, 050007-1-050007-9; https://doi.org/10.1063/5.0036897

Published by AIP Publishing. 978-0-7354-4057-9/\$30.00 


$$
\begin{gathered}
\operatorname{Gr}\left(V_{x} \frac{\partial V_{x}}{\partial x}+V_{y} \frac{\partial V_{x}}{\partial y}\right)=-\operatorname{Gr} \frac{\partial P}{\partial x}+\Delta^{*} V_{x}, \operatorname{Gr}\left(V_{x} \frac{\partial V_{y}}{\partial x}+V_{y} \frac{\partial V_{y}}{\partial y}\right)=-\operatorname{Gr} \frac{\partial P}{\partial y}+\Delta^{*} V_{y} \\
\frac{\partial P}{\partial z}=\frac{2 \delta V_{x}}{\operatorname{Gr} \cdot \operatorname{Ek}}+\frac{\delta}{\operatorname{Gr}} T, \operatorname{Ra}\left(V_{x} \frac{\partial T}{\partial x}+V_{y} \frac{\partial T}{\partial y}\right)=\Delta^{*} T \\
\frac{\partial V_{x}}{\partial x}+\frac{\partial V_{y}}{\partial y}=0
\end{gathered}
$$

where $V_{x}, V_{y}$ are the dimensionless components of the fluid velocity vector; the dimensionless horizontal coordinates $x$ and $y$ are determined by a characteristic length scale $L$; the transverse coordinate $z$ is determined by the fluid layer thickness $h ; \delta=h / L$ is the ratio of the length scales; $\vartheta$ is the difference between the maximum temperature and the minimum temperature, $T$ is dimensionless temperature; $v$ is the kinematic viscosity of the fluid; $\beta$ is the coefficient of volume expansion of the fluid; $g$ is the acceleration of free fall; Gr is the Grashof number; $R a$ is the Rayleigh number; $\mathrm{Ek}=v /(L \Omega)$ is the Ekman number; $\Omega$ is the angular velocity; $P$ is the pressure divided by the kinetic energy; the Laplace operator is as follows:

$$
\Delta^{*}=\frac{\partial^{2}}{\partial x^{2}}+\frac{\partial^{2}}{\partial y^{2}}+\frac{1}{\delta^{2}} \frac{\partial^{2}}{\partial z^{2}}
$$

The Coriolis force is parallel to the axis oz of the local coordinate system. The velocity vector of the fluid lies in the plane $o x y$.

For system (1), we set the following boundary conditions. On the lower surface of the fluid layer set by the equation $z=0$, the temperature is defined as a linear function of the horizontal coordinates

$$
\left.T\right|_{z=0}=A_{0}+x A_{1}+y A_{2}
$$

where $A_{0}$ is the value of the background temperature (can be assumed to be zero); $A_{1}, A_{2}$ are the constants. The noslip condition is also set on the lower surface of the fluid layer,

$$
\left.V_{x}\right|_{z=0}=0,\left.V_{y}\right|_{z=0}=0
$$

The upper free surface is assumed to be plane, and it is given by the equation $z=1$. It sets the temperature in the form similar to (2),

$$
\left.T\right|_{z=1}=B_{0}+x B_{1}+y B_{2}
$$

where $B_{0}$ is the value of the background temperature; $B_{1}, B_{2}$ are the constants.

On the free surface of the fluid, we consider the atmospheric pressure to be constant, independent of the longitudinal coordinates. We take the background value of the atmospheric pressure $\left.P_{0}\right|_{z=1}$ as a reference value and consider it equal to zero:

$$
\left.P_{0}\right|_{z=1}=0,\left.P_{1}\right|_{z=1}=0,\left.P_{2}\right|_{z=1}=0
$$

The Marangoni-Gibbs thermocapillary effect acts on the free surface. The Marangoni boundary condition in dimensionless variables takes the form

$$
\left.\left(\frac{\partial V_{x}}{\partial z}+\frac{\partial V_{z}}{\partial x}\right)\right|_{z=1}=-M g \frac{\partial T}{\partial x},\left.\left(\frac{\partial V_{y}}{\partial z}+\frac{\partial V_{z}}{\partial y}\right)\right|_{z=1}=-M g \frac{\partial T}{\partial y}
$$

where $M g$ is the dimensionless Marangoni number. 


\section{EXACT SOLUTION NEAR THE EQUATOR}

The exact solution will be found in the following form [8-15]:

$$
\begin{gathered}
V_{x}=U(z), V_{y}=V(z) \\
P=P_{0}(z)+x P_{1}(z)+y P_{2}(z), T=T_{0}(z)+x T_{1}(z)+y T_{2}(z) .
\end{gathered}
$$

Substituting the type of exact solution (7) in the original system of partial differential equations (1) and equating the coefficients for the same degrees of variables $x$ and $y$, we obtain a nonlinear system of ordinary differential equations

$$
\begin{gathered}
T_{1}^{\prime \prime}=0, T_{2}^{\prime \prime}=0, P_{1}^{\prime}=-\frac{\delta}{\mathrm{Gr}} T_{1}, P_{2}^{\prime}=-\frac{\delta}{\mathrm{Gr}} T_{2}, \\
U^{\prime \prime}=\mathrm{Gr} \delta^{2} P_{1}, V^{\prime \prime}=\mathrm{Gr} \delta^{2} P_{2} T_{0}^{\prime \prime}=\operatorname{Ra} \delta^{2}\left(T_{1} U+T_{2} V\right), \\
P_{0 T}^{\prime}=\frac{\delta T_{0}}{\mathrm{Gr}}, P_{0 U}^{\prime}=\frac{\delta}{\mathrm{Gr}} \frac{U}{\mathrm{Ek}}, P_{0}=P_{0 T}+P_{0 U} .
\end{gathered}
$$

The equations of system (8) are solved sequentially and written in the sequence of solving. The background pressure $P_{0}$ is divided into two terms. The term $P_{0 \mathrm{U}}$ is determined by the velocity component $U$ from system (8), and the term $P_{0 T}$ is determined by the background temperature $T$.

In view of the form of solution (7), the boundary conditions (2), (3), (4), and (5) will be written as

$$
\begin{gathered}
T_{1}(0)=A_{1}, T_{1}(1)=B_{1}, T_{2}(0)=A_{2}, T_{2}(1)=B_{2}, P_{1}(1)=0, P_{2}(1)=0, \\
U(0)=0, U^{\prime}(1)=-\delta M g T_{1}(1), V(0)=0, V^{\prime}(1)=-\delta M g T_{2}(1), \\
T_{0}(0)=A_{0}, T_{0}(1)=B_{0}, P_{0}(1)=0 .
\end{gathered}
$$

Taking into account that the desired functions depend only on the variable $z$, we denote by a prime the function derivatives with respect to $z$.

Under the given boundary conditions (9), the following polynomial solutions of system (8) determining the pressure and temperature gradients are obtained:

$$
\begin{gathered}
T_{1}=A_{1}+\left(B_{1}-A_{1}\right) z, T_{2}=A_{2}+\left(B_{2}-A_{2}\right) z \\
P_{1}=-\frac{\left(B_{1}\left(1-z^{2}\right)+A_{1}(z-1)^{2}\right) \delta}{2 \mathrm{Gr}}, P_{2}=-\frac{\left(B_{2}\left(1-z^{2}\right)+A_{2}(z-1)^{2}\right) \delta}{2 \mathrm{Gr}} .
\end{gathered}
$$

As in the case of middle latitudes, the temperature and pressure gradients are obtained as linear and quadratic functions of the variable $z$. The velocity vector components, in contrast to middle latitudes, are fourth-order polynomials with respect to $z$ :

$$
\begin{gathered}
U=\frac{z \delta^{3}}{24}\left[B_{1}\left(\left(z^{3}-6 z+8\right)-24 \frac{M g}{\delta^{2}}\right)-A_{1}\left(z^{3}-4 z^{2}+6 z-4\right)\right], \\
V=\frac{z \delta^{3}}{24}\left[B_{2}\left(\left(z^{3}-6 z+8\right)-24 \frac{M g}{\delta^{2}}\right)-A_{2}\left(z^{3}-4 z^{2}+6 z-4\right)\right] .
\end{gathered}
$$

As a result of integrating the last equations in system (8), taking into account the boundary conditions (9), we obtain the expressions for the background temperature and pressure 


$$
\begin{gathered}
T_{0}=A_{0}+z\left(B_{0}-A_{0}\right)+R a\left\{\left(B_{1}^{2}+B_{2}^{2}\right)\left(\frac{41 z \delta^{3}}{2520}-\frac{z^{4}\left(140-63 z+5 z^{3}\right) \delta^{3}}{5040}\right)+\right. \\
+\left(A_{1} B_{1}+A_{2} B_{2}\right) \delta^{3}\left(\frac{13 z}{504}-\frac{z^{3}\left(56-35 z+7 z^{3}-2 z^{4}\right)}{1008}\right)+\left(A_{1}^{2}+A_{2}^{2}\right) \delta^{3}\left[\frac{z}{126}-\frac{z^{3}\left(28-35 z+21 z^{2}-7 z^{3}+z^{4}\right)}{1008}\right]+ \\
\left.+M g \frac{z \delta}{12}\left[\left(A_{1} B_{1}+A_{2} B_{2}\right)\left(1+(z-2) z^{2}\right)+\left(B_{1}^{2}+B_{2}^{2}\right)\left(1-z^{3}\right)\right]\right\}, \\
P_{0 U}=-\frac{B_{1} M g\left(z^{2}-1\right) \delta^{2}}{2 E k G r}+\frac{B_{1}\left(-11+20 z^{2}-10 z^{3}+z^{5}\right) \delta^{4}}{120 E k G r}-\frac{A_{1}\left(4-10 z^{2}+10 z^{3}-5 z^{4}+z^{5}\right) \delta^{4}}{120 E k G r}, \\
P_{0 T}=-\frac{\left(B_{0}\left(1-z^{2}\right)+A_{0}(z-1)^{2}\right) \delta}{2 G r}+R a\left\{\frac{\left.\left(A_{1}^{2}+A_{2}^{2}\right)\left(11-32 z^{2}+56 z^{4}-56 z^{5}+28 z^{6}-8 z^{7}+z^{8}\right)\right) \delta^{4}}{8064 G r}-\right. \\
-\frac{\left.M g B_{1}+A_{2} B_{2}\right)\left(-21+52 z^{2}-56 z^{4}+28 z^{5}-4 z^{7}+z^{8}\right) \delta^{4}}{4032 G r}+\frac{\left(B_{1}^{2}+B_{2}^{2}\right)\left(183-328 z^{2}+224 z^{5}-84 z^{6}+5 z^{8}\right) \delta^{4}}{40320 G r}+ \\
\left.+\frac{M g \delta^{2}}{120 G r}\left[\left(A_{1} B_{1}+A_{2} B_{2}\right)\left(-2+5 z^{2}-5 z^{4}+2 z^{5}\right)-\left(B_{1}^{2}+B_{2}^{2}\right)\left(3-5 z^{2}+2 z^{5}\right)\right]\right\} .
\end{gathered}
$$

Note that the dynamic component depends on the components of the boundary temperature gradients linearly and that the temperature and the temperature term of the pressure are determined by quadratic forms of the components of the temperature gradients.

\section{ANALYSIS OF STAGNATION POINTS}

Stagnation points are the points on the $z$ axis where one of the velocity vector components becomes zero. Assume that the point where both velocity vector components become zero is termed a double stagnation point.

Thus, the search for stagnation points in this problem is reduced to determining the range of the system parameters at which the real roots of the polynomials (10) exist and lie on the length $(0,1)$. Since this problem considers the no-slip conditions on the lower boundary, there will always be a zero root of the polynomials (10).

Note that the polynomials (10) defining the solutions for $U$ and $V$ are identical in structure. Therefore, it is enough to examine one of them. The expression for the velocity component (10) is written as

$$
U=z \delta\left\{\frac{\delta^{2}}{24}\left[\left(B_{1}-A_{1}\right) z^{3}+4 A_{1} z^{2}-6 z\left(B_{1}+A_{1}\right)+4\left(2 B_{1}+A_{1}\right)\right]-B_{1} M g\right\}
$$

Expression (11) is a fourth-order polynomial, but one root is zero. Therefore, the search and study of stagnation points is reduced to the analysis of a third-order polynomial. First, let us examine the special case $A_{1}=B_{1}$. Expression (11) will take the form

$$
U=\frac{A_{1} z \delta^{3}}{6}\left[z^{2}-3 z+3\right]-z \delta A_{1} M g
$$

The analysis of the equation roots shows that one root will always lie outside the interval $(0,1)$, and the other falls inside the interval $(0,1)$ when the following condition is met:

$$
\frac{1}{6}<\frac{M g}{\delta^{2}}<\frac{1}{2}
$$


Therefore, there can be only one stagnation point.

Consider the general case $A_{1} \neq B_{1}$. Assume that $A_{1}>B_{1}$; the study of the existence of stagnation points is reduced to the study of the roots of the cubic polynomial

$$
g(z)=z^{3}-4 \mu z^{2}+6 z(2 \mu-1)-4(3 \mu-2)+(\mu-1) p,
$$

where, for brevity, $\mu=\frac{A_{1}}{A_{1}-B_{1}}, p=24 \frac{M g}{\delta^{2}}$.

Simple computations show that, when $\frac{3}{4}<\mu<\frac{3}{2}$, the derivative of the right-hand side of expression (12) with respect to $z$ has no real roots. Therefore, the function $g(z)$ is monotonic on the entire number line $z$, and there is either one stagnation point or none. When $\frac{1}{2}<\mu<\frac{3}{4}$, one zero of the derivative falls on the segment $(0,1)$. The second zero of the derivative cannot get into the segment $(0,1)$, and there cannot be more than two stagnation points.

Let us compare the signs of the function $g(z)$ at the ends of the segment $(0,1)$. To do this, we construct the function

$$
f(p, \mu)=g(0) \cdot g(1)=24-68 \mu+48 \mu^{2}+\left(11-27 \mu+16 \mu^{2}\right) p+(1-\mu)^{2} p^{2} .
$$

The equation $f(p, \mu)=0$ has the following roots with respect to $p$ :

$$
p_{1}=\frac{3-4 \mu}{\mu-1}, p_{2}=\frac{4(2-3 \mu)}{\mu-1} .
$$

Therefore, when $\frac{3-4 \mu}{\mu-1}<p<\frac{4(2-3 \mu)}{\mu-1}$, the function $f(p, \mu)$ is less than zero, and for all $\mu$ and $p$ satisfying this inequality, the function $g(z)$ has a single root on the segment $(0,1)$, the flow having a single stagnation point. If the function $f(p, \mu)$ is positive, the values of the function $g(z)$ at the ends of the segment $(0,1)$ are of the same sign. When $\mu \in\left[\frac{1}{2}, \frac{3}{4}\right]$, the derivative of the function $g(z)$ becomes zero at the point $z^{0} \in(0,1)$. Let us build the function $f_{d}(p, \mu)=g(0) \cdot g\left(z^{0}\right)$ having the form of a quadratic polynomial with respect to $p$. The roots of the equation $f_{d}(p, \mu)=0$ for the variable $p$ are

$$
p=\frac{-4(-2+3 \mu)}{-1+\mu}, \quad p=-\frac{4(-3+4 \mu)\left(18-21 \mu+8 \mu^{2}+(3-2 \mu) \sqrt{2} \sqrt{9-18 \mu+8 \mu^{2}}\right)}{27(-1+\mu)} .
$$

At $p$ lying in the interval

$$
-\frac{4(-3+4 \mu)\left(18-21 \mu+8 \mu^{2}+(3-2 \mu) \sqrt{2} \sqrt{9-18 \mu+8 \mu^{2}}\right)}{27(-1+\mu)}<p<\frac{-4(-2+3 \mu)}{-1+\mu},
$$

the function $f_{d}(p, \mu)$ takes negative values since the coefficient for the highest term is positive. Therefore, when the resulting inequality (13) is satisfied, taking into account that $p>0$, the function $g(z)$ has two roots on the segment $(0,1)$, and two stagnation points are possible.

The case $B_{1}>A_{1}$ is analyzed in a similar way. 
A double stagnation point can occur if the constants $A_{1}, A_{2}$, and $B_{1}, B_{2}$ are proportional. Moreover, if $A_{1} / A_{2}=B_{1} / B_{2}$, there is a double stagnation point shown in Fig. 3 and, if $A_{1} / A_{2}=-B_{1} / B_{2}$, there is a double stagnation point shown in Fig. 2.

The velocity profiles for different values of the temperature gradient are shown below. In the drawings of velocity profiles, the numbers indicate the following: 1 is the velocity component $U$; and 2 is the velocity component $V$; in the drawings of pressure profiles, the number 0 indicates the total pressure, $U$ is the dynamic term, and $T$ is the temperature term.

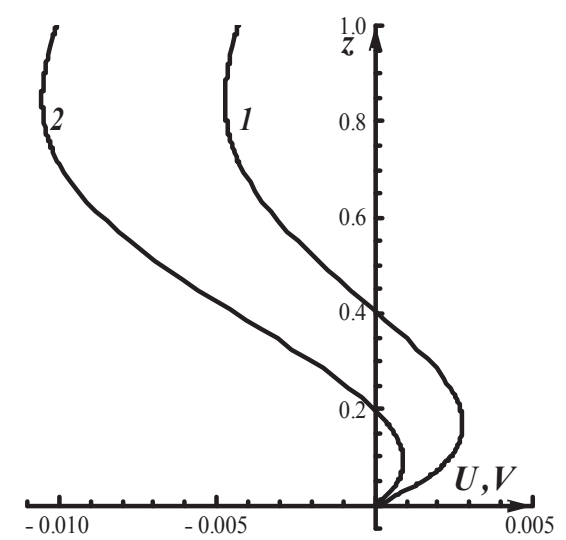

(a)

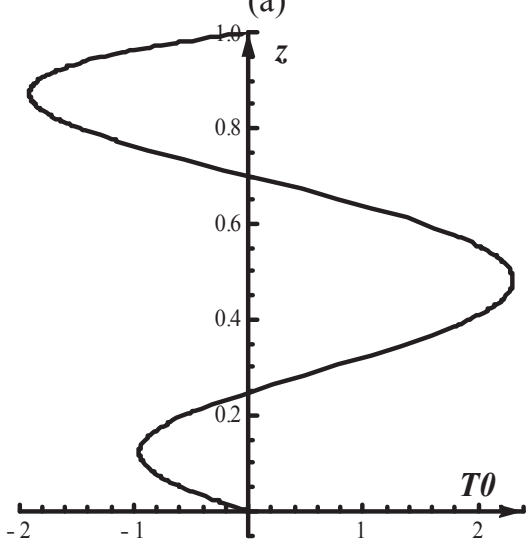

(c)

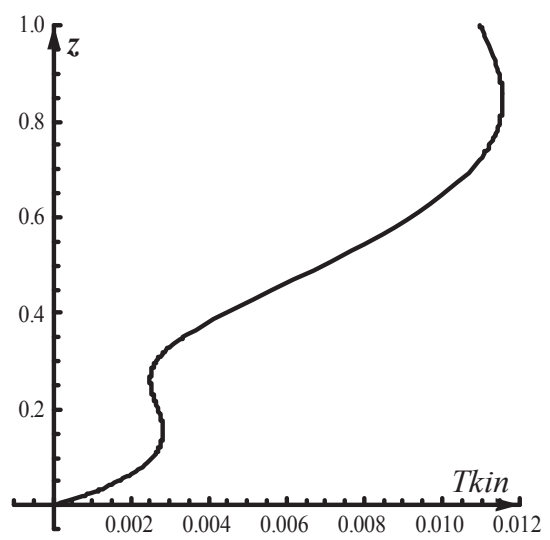

(b)

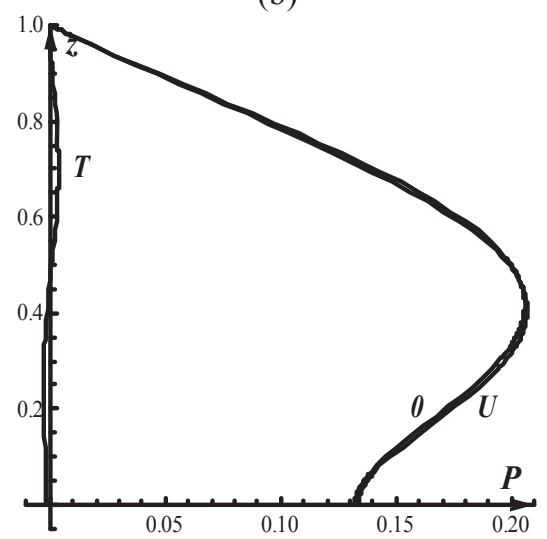

(d)

FIGURE 1. Velocity stagnation points: velocity profiles (a); kinetic energy profile (b); background temperature profile $T_{0}$ (c); $P_{U}, P_{T}, P_{0}$ pressure component profiles (d)

Figure 1 shows the case of two different velocity stagnation points. The flow is stratified by the temperature. The background pressure is determined by the dynamic term. The solution in Fig. 1 is computed with the following parameter values:

$$
\begin{aligned}
& A_{0}=0.0, B_{0}=0.0, A_{1}=1000.0, A_{2}=1000.0, B_{1}=-400.0, B_{2}=-450.0, \\
& \mathrm{Ek}=0.0001, \mathrm{Gr}=10.0, \mathrm{Ra}=50.0, M g=0.0001, \delta=0.1
\end{aligned}
$$

Figure 2 presents the case of a double stagnation point. At the same point, the kinetic energy becomes zero. The solutions are computed for the following parameter values:

$$
\begin{aligned}
& A_{0}=0.0, B_{0}=0.0, A_{1}=1000.0, A_{2}=500.0, B_{1}=-370.0, B_{2}=185.0, \\
& \mathrm{Ek}=0.0001, \mathrm{Gr}=10.0, \mathrm{Ra}=50.0, M g=0.0001, \delta=0.1
\end{aligned}
$$




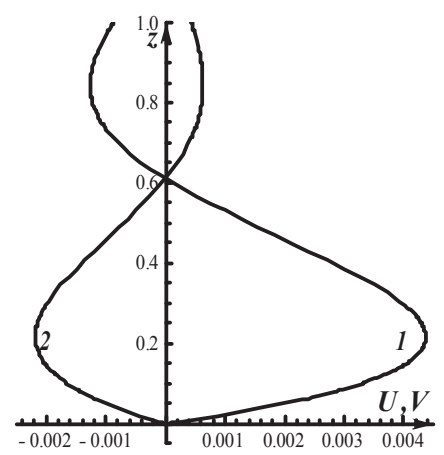

(a)

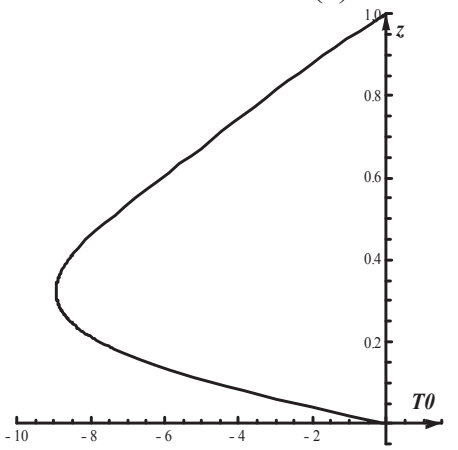

(c)

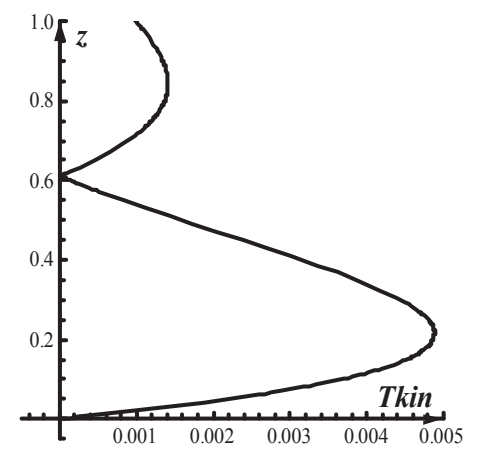

(b)

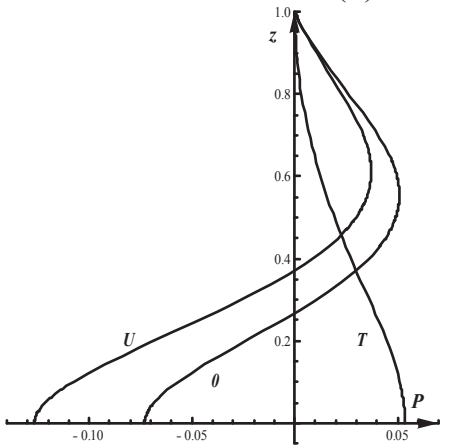

(d)

FIGURE 2. Double stagnation point: velocity profiles (a); kinetic energy profile (b); background temperature profile $T_{0}$ (c); $P_{U}, P_{T}, P_{0}$ pressure component profiles (d)

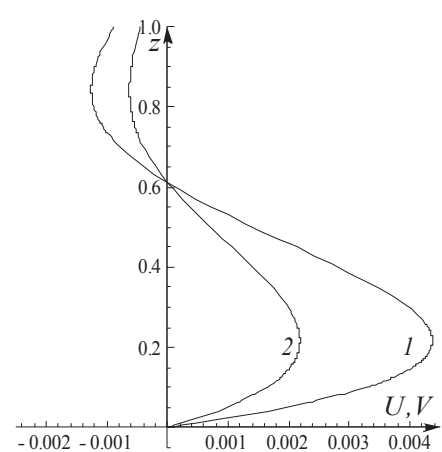

(a)

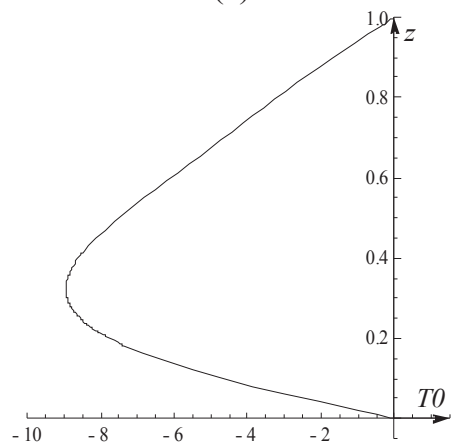

(c)

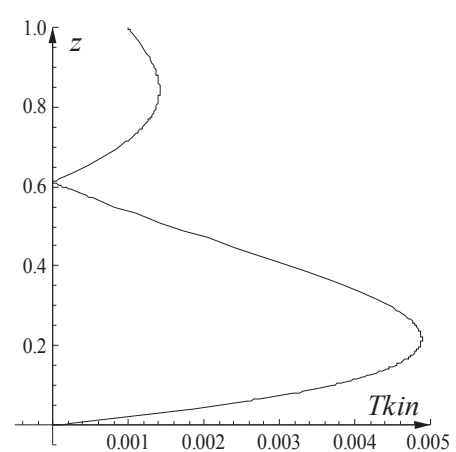

(b)

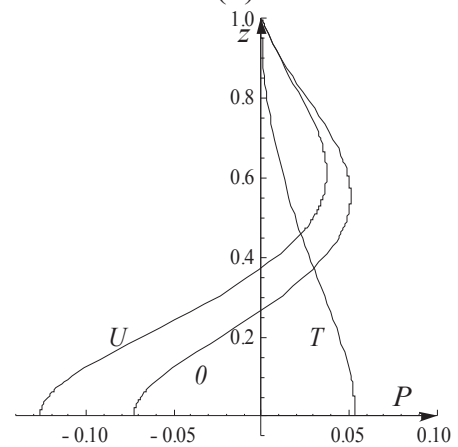

(d)

FIGURE 3. Solution with a different stagnation point: velocity profiles (a); kinetic energy profile (b); background temperature profile $T_{0}(\mathrm{c}) ; P_{U}, P_{T}, P_{0}$ pressure component profiles (d) 
Figure 3 shows a solution with a double velocity stagnation of a different type than that in Fig. 2. The solutions are computed for the following parameter values:

$$
\begin{aligned}
& A_{0}=0.0, B_{0}=0.0, A_{1}=1000.0, A_{2}=500.0, B_{1}=-370.0, B_{2}=-185.0, \\
& \mathrm{Ek}=0.0001, \mathrm{Gr}=10.0, \mathrm{Ra}=50.0, M g=0.0001, \delta=0.1
\end{aligned}
$$

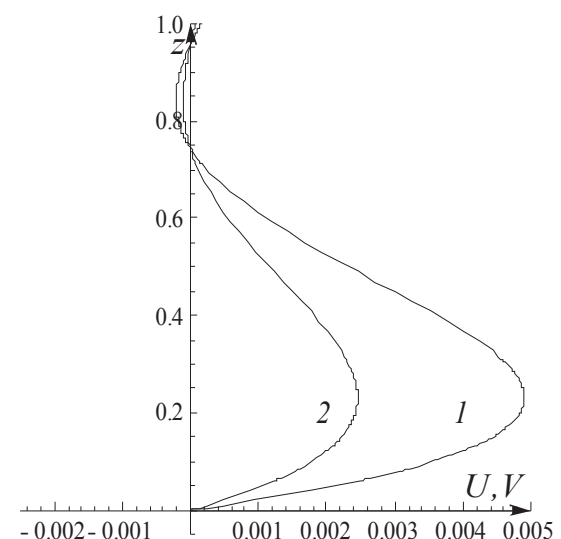

(a)

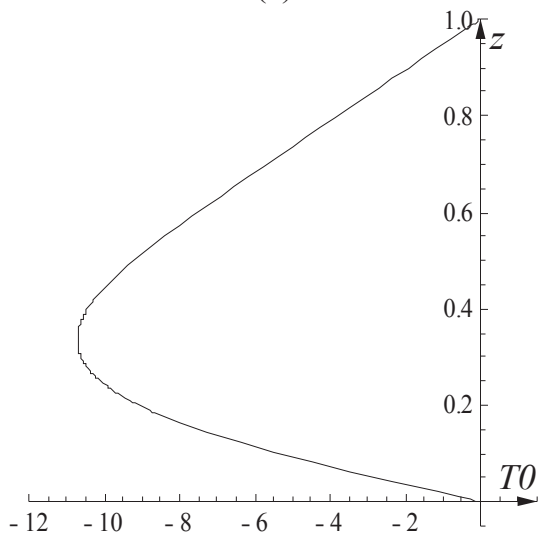

(c)

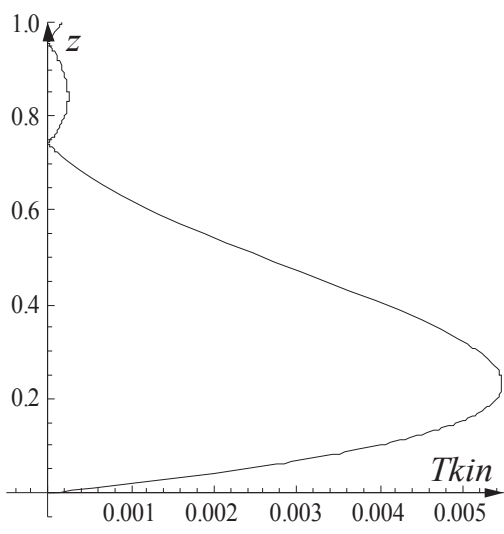

(b)

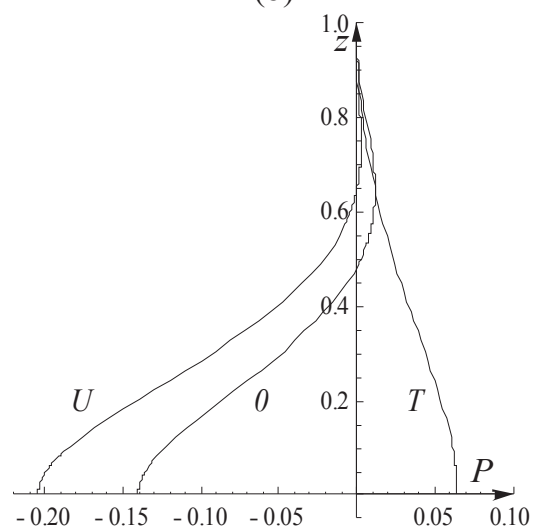

(d)

FIGURE 4. Two double stagnation points: velocity profiles (a); kinetic energy profile (b); background temperature profile $T_{0}$ (c); $P_{U}, P_{T}, P_{0}$ pressure component profiles (d)

Figure 4 presents the case of two double stagnant points. The kinetic energy becomes zero at two points. The solutions in Fig. 4 are computed for the following parameter values:

$$
\begin{aligned}
& A_{0}=0.0, B_{0}=0.0, A_{1}=1000.0, A_{2}=500.0, B_{1}=-361.0, B_{2}=-180.5, \\
& \mathrm{Ek}=0.0001, \mathrm{Gr}=10.0, \mathrm{Ra}=50.0, M g=0.0001, \delta=0.1
\end{aligned}
$$

\section{CONCLUSION}

An analytical solution for the convective flow of viscous incompressible fluid in the equatorial zone has been constructed with regard for two components of the Coriolis force. The solution for the equatorial zone, in contrast to the solution for the middle latitudes, has been constructed in the form of polynomials. The existence and position of stagnation points has been analyzed. The values of the parameters at which stagnation points can appear have been determined. The possible existence of a double stagnation point has been shown. 


\section{REFERENCES}

1. V. W. Ekman, Ark. Mat., Asrton. Fys. 2(11), 1-53, 1905.

2. A. I. Felzenbaum, Theoretical Bases and Methods for Calculating Steady Sea Currents (AN USSR, Moscow, 1960).

3. J. Pedlosky, Geophysical Fluid Dynamics (Springer-Verlag, New York, 1982).

4. G. K. Korotaev, E. N. Mikhaylova, and N. B. Shapiro, Theory of Equatorial Countercurrents in the World Ocean (Naukova Dumka, Kiev, 1986).

5. B. M. Boubnov and G. S. Golitsyn, Convection in Rotating Fluids (Kluwer Academic Publ., Dordrecht, 1995).

6. S. N. Aristov and K. G. Shvarts, Vortical Flows of the Advective Nature in a Rotating Fluid Layer (Perm State Univ., Perm, 2006).

7. S. N. Aristov and E.Yu. Prosviryakov, Theor. Found. Chem. Eng. 50(3), 294-301 (2016).

8. E. Yu. Prosviryakov, Theor. Found. Chem. Eng. 53(1), 112-120 (2019).

9. A. V. Gorshkov and E. Yu. Prosviryakov, Izvestiya, Atmospheric and Oceanic Physics 54(2), 189-195 (2018).

10. A. V. Gorshkov and E. Yu. Prosviryakov, AIP Conference Proceedings 1915, 040020 (2017).

11. A. V. Gorshkov and E. Yu. Prosviryakov, AIP Conference Proceedings 1915, 040019 (2017).

12. A. V. Gorshkov and E. Yu. Prosviryakov, AIP Conference Proceedings 2053, 040030 (2018).

13. A. V. Gorshkov and E. Yu. Prosviryakov, AIP Conference Proceedings 2053, 040029, (2018).

14. S. N. Aristov and E. Yu. Prosviryakov, Fluid Dynamics 10(2), 177-182 (2014).

15. S. N. Aristov and E. Yu. Prosviryakov, Fluid Dynamics 10(2), 25-31 (2016).

16. S. N. Aristov and K. G. Shvarts, Vortical Flows in Thin Fluid Layers (Vyatka State Univ., Kirov, 2011).

17. S. N. Aristov and K. G. Shvarts, J. of Applied Mechanics and Technical Physics 1, 216-223 (2016).

18. L. Kh. Ingel and S. N. Aristov. Institute of Experimental Meteorology 27(162), 142-157 (1996). 